\title{
Digitalisierung und Investitionsfinanzierung
}

\author{
Boris Augurzky und Andreas Beivers \\ () Der/die Autor(en) 2019 \\ J. Klauber et al. (Hrsg.), Krankenhaus-Report 2019 \\ https://doi.org/10.1007/978-3-662-58225-1_5
}

\section{Zusammenfassung}

Die geburtenstarken Jahrgänge werden schon bald aus dem Erwerbsleben ausscheiden. Der große „Rentenansturm" ist im Laufe der 2020er Jahre zu erwarten und mithin auch eine wachsende Nachfrage nach Gesundheitsleistungen. Gleichzeitig droht allerdings eine Rationierung auf dem Arbeitsmarkt für Gesundheitsberufe und infolgedessen auch der Leistungen für Patienten. Zentrale Aufgabe wird es daher sein, mit weniger Fachkräften mehr hilfsbedürftige Menschen zu versorgen, ohne dass die Arbeitsbelastung dieser Fachkräfte so sehr steigt, dass sie am Ende das Interesse am Gesundheitswesen verlieren und in anderen Branchen attraktivere Tätigkeiten suchen. Der Fokus muss daher auf arbeitssparendem technischem Fortschritt liegen. Dabei ist die unternehmerische Gestaltungsfreiheit ein zentrales Element, um medizinischen und besonders technologischen Innovationen Zugang zum Gesundheitswesen zu ermöglichen. Die anstehenden gewaltigen Herausforderungen im Gesundheitswesen werden nämlich nicht allein dadurch gemeistert werden können, dass die Ablauf- und Aufbauorganisation der einzelnen Leistungserbringer immer weiter optimiert werden. Vielmehr werden dazu auch völlig neue effizienzsteigernde Innovationen nötig sein, wie Digitalisierung der Medizin, Telemedizin, künstliche Intelligenz oder auch Robotik. Da sie Zeit bis zur Marktreife brauchen, gilt es, die Digitalisierung im Gesundheitswesen schon jetzt voranzutreiben. Länder wie Dänemark können hierbei gute Vorbilder sein. So hat die Digitalisierung dort u. a. in deren Krankenhausreform einen besonderen Stellenwert. Auch in Deutschland braucht es eine breit angelegte Digitalisierungsstrategie. Die Autoren schlagen daher einen „Digital Boost" vor, der Investitionen im Krankenhausbereich in die Digitalisierung durch ein zeitlich befristetes Investitionsprogramm von acht Jahren vorsieht. Dessen Ziel ist es, eine zeitgemäße IT-Infrastruktur und die elektronische Vernetzung der Krankenhäuser zu ermöglichen, wie sie in manch anderen Ländern bereits existiert.

The baby boomers will soon retire from working life. The large "pension rush" is to be expected in the course of the 2020s and thus a growing demand for health services. At the same time, however, there is a threat of rationing on the labour market for health care professions and, as a result, for services for patients. The central task will be to provide more people in need with fewer specialists without increasing the workload of these specialists to such an extent that in the end they lose interest in health care and look for more attractive jobs in other sectors. The focus must therefore be on labour-saving technological progress. Entrepreneurial freedom is a central element in implementing medical and especially technological innovations in the health care system. The enormous challenges facing the healthcare system cannot be mastered by simply optimising the workflow and organisational structure of the individual service providers. In fact, completely new efficiency-enhancing innovations such as digitalisation of medicine, telemedicine, artificial intelligence and robotics will be necessary. Since they need time to reach market maturity, it is important to push ahead with digitisation in the 
healthcare sector now. Countries like Denmark can serve as role models in this respect, since digitisation plays a significant role in its hospital reform. A broad-based digitisation strategy is also needed in Germany. The authors therefore propose a "digital boost" which provides for investments in digitisation in the hospital sector by means of an investment programme of eight years. Its aim is a modern IT infrastructure and electronic networking of hospital as it already exists in many other countries.

\subsection{Ausgangslage: Disruption durch Digitalisierung? ${ }^{1}$}

Derzeit befassen sich viele Akteure im Gesundheitswesen mit der Digitalisierung und diskutieren intensiv über die sich daraus ergebenden Chancen und Risiken. Den großen Chancen u. a. bei der Neudefinition von digitalen Prozessen und der Unterstützung von Ärzten und Pflegekräften durch künstliche Intelligenz stehen Ängste gegenüber: Nicht nur in Bezug auf die Datensicherheit, sondern auch hinsichtlich der Frage, wie sich die Sozialversicherung im digitalen Zeitalter aus Arbeitseinkommen finanzieren lässt. Übernehmen zukünftig Maschinen und künstliche Intelligenz vermehrt Aufgaben von Menschen und somit auch deren Arbeitsplätze, so erfolgt eine noch nie dagewesene Substitution von Humankapital - quer über alle Bildungsschichten - durch den Produktionsfaktor „Realkapital“'. Dies hätte schwerwiegende Folgen für die Finanzierungsgrundlage der GKV - der Grundlohnsumme. Demgegenüber ist der seit Jahren diskutierte demografische Wandel vermutlich geradezu harmlos. Die bis dato bestehende Finanzierungslogik der GKV war eine Antwort aus dem 19. Jahrhundert auf die „industrielle Revolution 1.0", die der Gesellschaft eine Beantwortung sozialpolitischer Fragen aufzwang.

So wird das Sozialversicherungssystem heute im Wesentlichen von der Produktivität ihrer Menschen, bzw. konkret das der sozialversicherungspflichtig Beschäftigten getragen. Das laufende Jahrzehnt ist dabei weitgehend durch eine Phase hoher wirtschaftlicher Aktivität und geringer Arbeitslosigkeit geprägt. Der Sachverständigenrat zur Begutachtung der gesamtwirtschaftlichen Entwicklung spricht in seinem Gutachten aus 2017 davon, dass sich die deutsche Wirtschaft in einem kräftigen Aufschwung befindet (SVR 2017). Er rechnet mit

1 Siehe auch Augurzky 2018.
Zuwachsraten des Bruttoinlandsprodukts von 2,0 Prozent im Jahr 2017 und von 2,2 Prozent im Jahr 2018 und spricht gar von einer Überauslastung der deutschen Wirtschaft. Aufgrund ihrer großen Zahl haben die geburtenstarken Jahrgänge aus den Jahren 1955 bis 1969 dabei einen gewichtigen Anteil. Die höchste Anzahl an Geburten wurde 1964 mit 1.357.304 (gesamtdeutsche Zahlen) erreicht. Im Jahr 1972 fiel die Anzahl erstmals deutlich unter eine Million und am niedrigsten lag sie 2011 mit rund 663.000 .

Die geburtenstarken Jahrgänge werden jedoch schon bald aus dem Erwerbsleben ausscheiden. Im Jahr 2018 sind die ältesten „Babyboomer“ 63 Jahre alt, spätestens 2020 werden sie in Rente gehen. Der große „Rentenansturm“ ist im Laufe der 2020er Jahre zu erwarten. Der jüngste Babyboomer wird etwa 2034 in Rente gehen. Mithin steigt damit die Zahl der zu versorgenden Rentner und ebenso altersbedingt die Zahl der Patienten. Im Gegenzug kommen weit weniger jüngere Menschen nach, welche die Arbeit der ausscheidenden Babyboomer übernehmen könnten, sodass mit einem weiter wachsenden Engpass an Fachkräften auf dem Arbeitsmarkt zu rechnen ist (Augurzky 2018).

Während also der Versorgungsbedarf über alle Sektoren hinweg in Deutschland steigen wird, können die dafür nötigen personellen Ressourcen nicht ohne Weiteres in gleichem Maß aufgebaut werden. Dazu müssten massiv Arbeitskräfte aus dem Ausland oder aus anderen Branchen für die $\mathrm{Ge}$ sundheitsbranche gewonnen werden, was sehr wettbewerbsfähige Arbeitsplätze verlangt, also u. a. steigende Löhne. Da im deutschen Gesundheitswesen die Preise für Gesundheitsleistungen staatlich reguliert sind, können höhere Löhne jedoch nicht einfach über höhere Preise der Leistungserbringer aufgefangen werden. Wenn die Löhne damit nicht ausreichend steigen, droht eine Rationierung auf dem Arbeitsmarkt für Gesundheitsberufe und infolgedessen auch der Leistungen für Patienten. In 
vielen ländlichen Regionen, die neben der Bevölkerungsalterung auch mit dem Wegzug von Menschen zu kämpfen haben, dürfte sich diese Problematik noch verschärfen.

Grundsätzlich gibt es drei Wege, diesem Problem zu begegnen. Der erste Weg besteht darin, das Nachfragewachstum nach Gesundheitsleistungen durch geeignete Maßnahmen zu reduzieren. Der zweite Weg geht über die Erhöhung der Produktivität und beim dritten Weg würden schlichtweg mehr Ressourcen für das Gesundheitswesen zur Verfügung gestellt, z. B. durch Erhöhung der Beiträge der Sozialversicherungen. Da der letztgenannte Weg anderweitige Nachteile mit sich bringt, wie zum Beispiel die Verteuerung des knappen Faktors Arbeit, kann er sicherlich nicht der einzig zu beschreitende Weg sein, auch wenn er für die Politik „schmerzfreier" wäre. Insofern müssen auch die ersten beiden Wege ernsthaft in Erwägung gezogen werden. Zentrale Aufgabe wird es dabei sein, mit weniger Fachkräften mehr hilfsbedürftige Menschen zu versorgen, ohne dass die Arbeitsbelastung dieser Fachkräfte so sehr steigt, dass sie am Ende das Interesse am Gesundheitswesen verlieren und in anderen Branchen attraktivere Tätigkeiten suchen.

Der Fokus muss also auf arbeitssparendem technischem Fortschritt liegen. Das bedeutet, medizinisch-technischer Fortschritt im Gesundheitswesen darf künftig nicht allein dazu führen, dass entweder bestehende Leistungen teurer angeboten oder zusätzliche Leistungen ermöglicht werden. Er muss vielmehr so geartet sein, dass es gelingt, den Personaleinsatz je Patient reduzieren zu können, unter der Nebenbedingung konstanter oder sogar steigender Behandlungs- bzw. Betreuungsqualität. Die inzwischen viel gerühmte "Digitalisierung" hätte das Potenzial dazu. Wie eingangs geschildert, gibt es sogar Befürchtungen, dass mit der Digitalisierung generell viele menschliche Tätigkeiten entfallen und dass daher viele Menschen in der Zukunft keine Arbeit mehr finden. Zum Beispiel könnten selbstfahrende Pkw dazu führen, dass der Beruf des Taxifahrers ausstirbt. Die Digitalisierung des Finanzsektors könnte den Beruf des Bankers gefährden und generell könnte künstliche Intelligenz (KI) vermehrt menschliche Tätigkeiten übernehmen - auch komplexere. Tatsächlich werden schon heute einfache
Sportnachrichten von Computern geschrieben (Augurzky 2018).

Übersehen wird dabei aber, dass gleichzeitig neue Berufe entstehen, zum Beispiel Berufe rund um die Entwicklung und Wartung dieser neuen Technologien oder für neue Dienstleistungen, die heute noch gar nicht existieren. Arbeitskräfte, deren Branche von der Digitalisierung "betroffen“ ist, könnten - zumindest mittelfristig - in Branchen wechseln, die weiterhin einen Bedarf an menschlicher Arbeitskraft haben, wie beispielsweise das Gesundheitswesen. Denn insbesondere in diesem Bereich besteht nicht nur eine große Nachfrage, sondern auch ein spezieller Bedarf an menschlicher Empathie, welcher nur partiell durch Digitalisierung substituiert werden kann. Es ist noch nicht lange her, da waren über 90 Prozent der Bevölkerung in der Landwirtschaft tätig. Mit der industriellen Revolution im 19. Jahrhundert änderte sich dies. Heutzutage arbeiten unter 5 Prozent der Menschen in der Landwirtschaft. Das bedeutet aber nicht, dass nunmehr 85 Prozent der Bevölkerung arbeitslos wären. Völlig neue Technologien und Produkte und damit verbundene Tätigkeiten sind im Laufe der Zeit entstanden. Die Nachfrage nach Arbeitskräften hat nicht nachgelassen, auch weil die Wünsche der Menschen fast grenzenlos sind. Tatsächlich bietet der Eintritt in das Zeitalter der Digitalisierung in Deutschland die große Chance im genau richtigen Moment, die anstehende demografische Herausforderung zu meistern und damit Rationierung von Leistungen in der Gesundheitsversorgung vermeiden zu können. Wichtig ist dabei nur, dass die Politik rechtzeitig die Herausforderungen erkennt, die regulatorischen Rahmenbedingungen entsprechend setzt und die Bevölkerung mitnimmt, statt sie mit ihren Ängsten und Befürchtungen allein zu lassen.

Hilfreich für das stark regulierte Gesundheitswesen wird dabei die durch finanzielle und personelle Ressourcenknappheit entstehende Notsituation sein, die im Laufe der 2020er Jahre entstehen wird. Denn solange die Kassen wie derzeit gefüllt sind, wird das Beharrungsvermögen des Gesundheitswesens mitsamt seinen bestehenden Ineffizienzen obsiegen, weil bestehende Besitzstände finanziell bedient werden können. Relevante Veränderungen, gerade in einem regulierten System, lassen sich nur erreichen, 
wenn die Ressourcen so knapp werden, dass heilige Kühe geschlachtet und alte Zöpfe aus der Not heraus abgeschnitten werden müssen. Plötzlich wird gehen, was bislang regulatorisch ausgeschlossen war, und es wird gelten „Pragmatismus vor Rationierung“. Durch eine gezielte, staatliche geförderte Investitionslenkung im Bereich der Digitalisierung der Gesundheitswirtschaft können jedoch schon heute wichtige Grundlagen dafür gelegt werden.

\subsection{Digitalisierung als eine Lösungsoption für das deutsche Gesundheitswesen}

Unternehmerische Gestaltungsfreiheit ist wichtig, um medizinischen und besonders technologischen Innovationen den Zugang zum Gesundheitswesen zu ermöglichen. Die anstehenden gewaltigen Herausforderungen im Gesundheitswesen im Laufe der 2020er Jahre werden nicht allein dadurch gemeistert werden können, dass die Ablauf- und Aufbauorganisation der einzelnen Leistungserbringer immer weiter optimiert werden. Vielmehr werden dazu auch völlig neue effizienzsteigernde und möglicherweise systemverändernde Innovationen nötig sein. Darunter fallen Themen wie Digitalisierung der Medizin, Telemedizin, künstliche Intelligenz, Robotik und Gendiagnostik, aber auch die Ambulantisierung der Medizin (Augurzky et al. 2018a).

Daher gilt es, die Digitalisierung im Gesundheitswesen schon jetzt voranzutreiben, um die erwartete unangenehme Notsituation in den 2020er Jahren zeitlich möglichst kurz zu halten. Denn die Digitalisierung wird sich, wenn es so weit ist, nicht per Knopfdruck scharf schalten lassen. Digitalisierung ist äußerst vielschichtig und erfordert eine Disruption vieler bestehender Systeme. In der Ökonomie ist dieses Phänomen gemäß dem österreichischen Volkswirtschaftler und Sozialwissenschaftler Joseph A. Schumpeter auch als „schöpferische Zerstörung" bekannt, wonach jede relevante (ökonomische) Entwicklung auf dem Prozess der schöpferischen bzw. kreativen Zerstörung beruht. So werden durch eine erfolgreiche Neukombination von Produktionsfaktoren alte Strukturen verdrängt und damit eine Neuordnung zugelassen (Schumpeter 2018). Um diese digitale Neuordnung im Gesund- heitswesen zu erreichen, werden Jahre, eher sogar ein ganzes Jahrzehnt vergehen. Die Digitalisierung im Gesundheitswesen setzt dabei an verschiedenen Stellen an (• Abb. 5.1) und muss zahlreiche Hürden überspringen. Grundsätzlichen sollen hier drei Arten von Digitalisierung unterschieden werden.

Erstens geht es um innerbetriebliche Prozesse von Leistungserbringern. Ihre Digitalisierung ist ureigene Aufgabe der Leistungserbringer und sollte von ihnen umgesetzt werden, sofern sie unter dem Kosten-Nutzen-Aspekt für sie vorteilhaft ist. Zweitens geht es um die Digitalisierung von Leistungen vornehmlich zwischen Leistungserbringern und Patienten (B2C), aber auch zwischen verschiedenen Leistungserbringern (B2B). Darunter fallen Themenfelder wie Sensorik, Ambient Assisted Living (AAL), Wearables, Insidables, Virtual Reality, Augmented Reality, Telemedizin, Apps, künstliche Intelligenz, Spracherkennung und Robotik. In der Regel dürften sie in den privatwirtschaftlichen Bereich fallen.

An dritter Stelle folgt die Digitalisierung der Vernetzung der Akteure des Gesundheitswesens inklusive der Patienten. Kern dieser Vernetzung bildet die elektronische Patientenakte (EPA). Im Idealfall ist sie die strukturierte und standardisierte Sammelstelle für sämtliche Patientendaten und bietet $\mathrm{zu}$ gleich ein Zugriffs- und Kontrollsystem (Lux und Breil 2017). Da zu erwarten ist, dass die elektronische Patientenakte mit standardisierter Telematikinfrastruktur zwar vielfältige positive Effekte für das Gesundheitssystem bewirkt (Amelung et al. 2016), aus denen aber der Entwickler der Patientenakte selbst keine Vorteile zieht, sollte ihre Entwicklung und Implementierung eine öffentliche Aufgabe sein.

Die Potenziale einer standardisierten digitalen und ganzheitlichen Erfassung der Gesundheitsdaten in Form einer elektronischen Patientenakte sind groß. Wenn jeder Leistungserbringer über einen vom Patienten autorisierten Zugang zur EPA die individuelle Krankheitsgeschichte abrufen kann, spart er selbst nicht nur Zeit, weil die Vorgeschichte nicht erst erfragt werden muss, sondern es können auch Doppeluntersuchungen vermieden werden. Hinzu kommen generell Potenziale im Bereich der Prozessoptimierung der Leistungserbringung, wenn viele Abfragen automatisiert erfolgen können und innerund besonders außerbetriebliche Schnittstellen genormt sind. Darüber hinaus kann mit der EPA die 


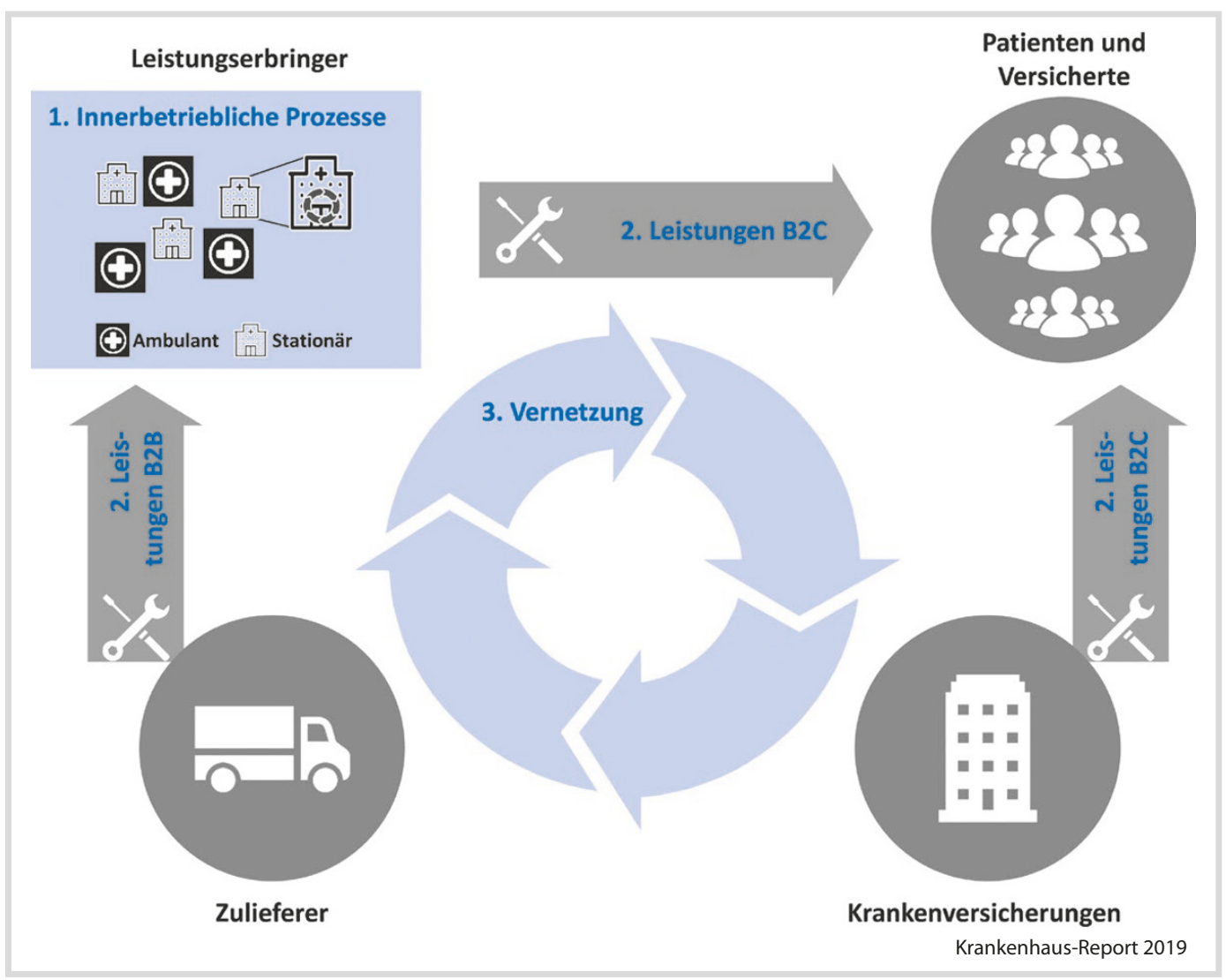

- Abb. 5.1 Arten der Digitalisierung (Quelle: Augurzky 2018; Copyright: W. Kohlhammer GmbH, mit freundlicher Genehmigung)

Diagnosestellung unterstützt werden - idealerweise sogar durch automatisierte Verknüpfung der Patientendaten mit bestehenden medizinischen Datenbanken. Bei einer ganzheitlichen und damit insbesondere sektorenübergreifenden Datenerfassung kann der Therapieverlauf einschließlich der Medikation verfolgt werden. Ergänzend können vom Patienten über Wearables, Insidables oder Apps selbst erhobene Gesundheitsdaten eingespeist werden, sodass die erhobenen Zeitreihen viel dichter werden, als dies mit nur wenigen Messpunkten während eines Arztbesuchs jemals möglich ist.

Besonders profitieren würde auch die Versorgungsforschung. Mittelfristig lassen sich damit erfolgreiche von erfolglosen Behandlungspfaden trennen. Die knappen Ressourcen des Gesundheitssystems könnten dann in die erwiesenermaßen erfolgreichen Pfade gelenkt werden, sodass sich insge- samt das Kosten-Nutzen-Verhältnis der eingesetzten Ressourcen spürbar verbessern würde. Durch geeignete Apps, die auf die Daten der Patientenakte zugreifen, könnte die Compliance der Patienten erhöht werden, zum Beispiel durch schlichte Erinnerungsfunktionen und Monitoring oder ggf. sogar durch digital gestützte Therapien, wie sie heute vereinzelt schon existieren. Mithin würde die Transparenz über das Versorgungsgeschehen deutlich gesteigert. Aber selbst ohne solche Potenziale wird sich der Bürger über kurz oder lang die Frage stellen, weshalb er im digitalen Zeitalter nicht über mobile Endgeräte auf seine persönlichen Gesundheitsdaten zugreifen kann, wie er es aus anderen Branchen gewohnt und wie es in anderen Ländern heute schon möglich ist.

In der wachsenden Transparenz kann allerdings auch eine Gefahr liegen, worauf Rebitschek, 

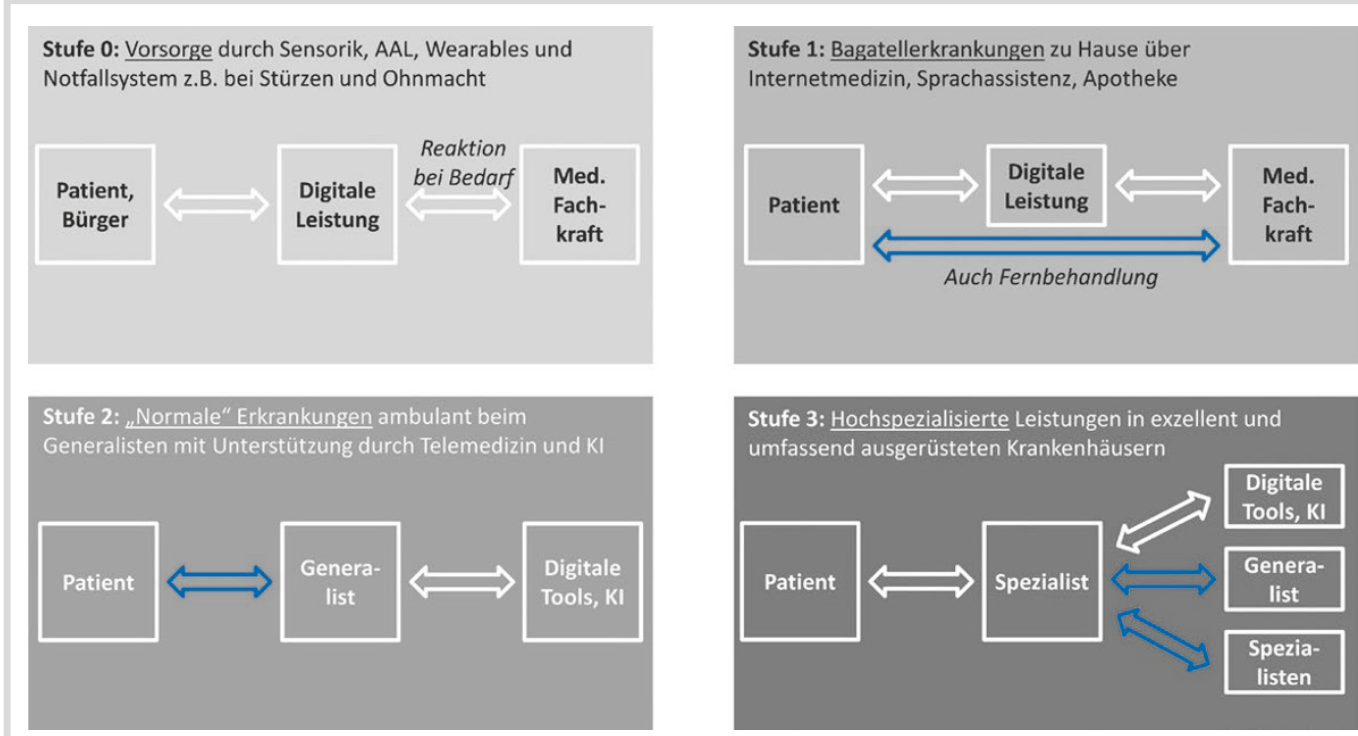

Krankenhaus-Report 2019

Abb. 5.2 Mögliche Versorgungsstruktur der Zukunft (Quelle: Augurzky 2018 angelehnt an DIV-Report 2017; Copyright: W. Kohlhammer $\mathrm{GmbH}$, mit freundlicher Genehmigung)

Gigerenzer und Wagner (2017) hinweisen. Wenn der Leistungserbringer aufgrund der allgegenwärtigen Transparenz damit rechnen muss, dass jeder Schritt, der sich hinterher als falsch herausstellt, zu einem Haftungsproblem werden kann, wird er auf „Defensivmedizin“ umschwenken. D. h. er wird genau abwägen, ob er einer erfolgversprechenden, aber risikoreichen Behandlung eine weniger erfolgversprechende, aber sichere Behandlung vorzieht. Die durchschnittliche Versorgungsqualität könnte dadurch sinken. Hinzu kommt die Gefahr des „Datenraubs“. Sie ist zwar im digitalen Zeitalter nicht neu. Der Schaden eines solchen Datenraubs ist jedoch weitaus höher, weil große Datenmengen auf einmal abhandenkommen können.

Die Digitalisierung des Gesundheitswesens besitzt darüber hinaus jedoch auch die Chance, wirtschaftlichere Angebotsstrukturen zu schaffen. Die Patientensteuerung kann aufgrund der fundierten Informationen über den Patienten weitaus effektiver geschehen als im heutigen System. Mithin kann der Versorgungsbedarf des Patienten besser dem Versorgungsangebot zugeordnet werden. - Abb. 5.2 stellt eine mögliche Versorgungsstruktur bei einem durchgehend digitalisierten Gesundheitswesen dar
(DIV-Report 2017). In der Stufe 0 geht es um die Überwachung von Gesundheit, die sich vollständig auf automatisierte Funktionen stützen würde, ergänzt um künstliche Intelligenz. Der Patient oder Bürger würde sich passiv verhalten. Menschliche Eingriffe wären nur bei Auffälligkeiten nötig, wenn Grund zur Annahme besteht, dass Hilfe erforderlich ist. Stufe 0 wäre aufgrund der Automatisierung arbeitssparend. In Stufe 1 würde der Patient oder Bürger selbst aktiv, wenn er Unregelmäßigkeiten in seinem Gesundheitszustand wahrnimmt, die allerdings nicht bedrohlich sind („Bagatellerkrankungen"). Er würde sich zum Beispiel an seinen sprachbegabten, durch künstliche Intelligenz ertüchtigten Roboter im eigenen Wohnzimmer wenden. Bei Bedarf kann er eine Verbindung zu einer medizinischen Fachkraft herstellen. Sollte eine einfache körperliche Untersuchung oder z. B. eine Blut- oder Urinprobe zusätzlich erforderlich sein, kann der Patient auf Anraten der Fachkraft z. B. die nächstgelegene Apotheke aufsuchen. ${ }^{2}$

2 In der Schweiz baut Medgate derzeit ein solches telefonund apothekenbasiertes System auf. Siehe „Mini Clinic" auf www.medgate.ch. 
Bei „normalen“ bzw. einfacheren Einmal- oder chronischen Erkrankungen sucht der Patient den ärztlichen Generalisten auf, der intensiv auf digitale Tools wie die EPA, vernetzte Datenbanken und künstliche Intelligenz zurückgreift (Stufe 2). Anders als in Stufe 1 gibt es hier also einen Coach für den Patienten, der ihn betreut und ihm Erkenntnisse aus Datenanalysen, der weltweiten medizinischen Literatur und digitalen Tools erläutert. In Stufe 3 finden sich Patienten mit komplexen Erkrankungen, die hochspezialisierte Leistungen benötigen. Sie stehen in direktem Kontakt mit einem entsprechenden Spezialisten, der wiederum andere Spezialisten zu Rate ziehen kann und der außerdem im Austausch mit dem betreuenden Generalisten steht und wie in Stufe 2 auf sämtliche digitale Tools weltweit zurückgreifen kann. In der Realität wird es hierbei noch weitere Abstufungen geben können, und es bedarf einer effektiven Patientensteuerung, um den richtigen Zugang zu den Versorgungsstufen zu gewährleisten.

\subsection{Das smarte Krankenhaus 4.0: Anspruch und Wirklichkeit}

Die Kritik am deutschen Gesundheitswesen in Bezug auf dessen mangelnden Grad an Digitalisierung (s. zum Beispiel Amelung et al. 2016) gilt gerade auch für Krankenhäuser. Betrachtet man den Digitalisierungsgrad der deutschen Krankenhauslandschaft, zeigt sich ein durchwachsenes Bild. Zwar ist IT im Krankenhaus schon seit einem halben Jahrhundert im Einsatz, anfangs jedoch nur für die Verwaltung. Die Architektur der alten Systeme war fall- und abrechnungsbezogen aufgebaut, was aus heutiger Sicht eine falsche Ausrichtung war. Denn heute ist ein übergreifender Patientenbezug nötig und gewünscht, nicht nur ein Fallbezug. In den 1990er und frühen 2000er Jahren wurden die KISSysteme (Krankenhausinformationssystem) immer leistungsfähiger. IT wurde nun genutzt, um Abläufe $\mathrm{zu}$ verbessern und zu koordinieren. Es entstand eine Workflow-Unterstützung der IT. Mit der IT-Workflow-Unterstützung wurde es für das Personal leichter, OPs zu planen und durchzuführen. Damit war eine effizientere Steuerung mit weniger Arbeitsaufwand möglich. Aber dafür wurden auch neue KIS-Systeme notwendig, mit neu designter Systemarchitektur.

Als nächstes fasste die Vernetzung im Krankenhaus Fuß, anfangs in Form eines Datenaustauschs zwischen fest installierten Rechnern auf Station oder in Arztzimmern, später in Form von mobilen Lösungen, die den Zugriff auf die Patientenakte von jedem Ort im Krankenhaus ermöglichten. Genau an dieser Stelle ergab sich das ursprüngliche Problem für die gegenwärtige Situation (Kellermann 2018). Zwar erlauben IT-Anwendungen mittlerweile die komplette Vernetzung, aber nicht in jedem Krankenhaus hat sich beispielsweise eine elektronische Patientenakte als Grundlage für Vernetzung durchgesetzt. Außerdem macht sich der Mangel an Investitionsmittel und das knappe Personal für den IT-Bereich im Krankenhaus bemerkbar.

Obwohl der Gesetzgeber sowohl durch das EHealth-Gesetz (Gesetz für sichere digitale Kommunikation und Anwendungen im Gesundheitswesen) als auch durch den Koalitionsvertrag aus dem Jahr 2018 dem Thema „Digitalisierung im Gesundheitswesen“ große Aufmerksamkeit schenkt, sind viele Krankenhäuser zurückhaltend bei der Umsetzung. So haben viele deutsche Krankenhäuser keine wirkliche Digitalisierungsstrategie und somit ist u. a. E-Health oftmals noch kein Managementthema (Kellermann 2018). Dies spiegelt sich auch im ITReport Gesundheitswesen 2018 wider (Hübner et al. 2018). An der Studie nahmen 224 IT-Leitungen deutscher Krankenhäuser teil. Von den Befragten gaben lediglich 37 Prozent an, dass sie einen IT-Plan haben, der in den strategischen Krankenhausplan integriert ist. Nur knapp die Hälfte der Studienteilnehmer hat ein getrenntes Budget für IT-Aufwendungen und IT-Investitionen. 23 Prozent haben keine Trennung zwischen Budget, welches sie für IT verwenden, und Budget für andere Investitionen. Dabei hat ein Großteil der Befragten eine eigene ITAbteilung im Haus mit im Durchschnitt sieben Mitarbeitern. Allerdings ist der IT-Leiter nicht in die Krankenhausleitung integriert. In 35 Prozent der Häuser übernimmt eine Pflegekraft die Leitung der IT. Weiteres Ergebnis der Studie ist, dass knapp 80 Prozent der Krankenhäuser keine nach ISO (International Organization for Standardization) oder KTQ (Kooperation für Transparenz und Qualität) zertifizierte IT-Abteilung haben (Hübner et al. 2018). 
Da die Digitalisierung in einem Krankenhausunternehmen ein groß angelegtes Projekt ist, sind dafür enorme Investitionen notwendig. Die Knappheit der Investitionsfördermittel für Krankenhäuser erschwert es aber, solche Großprojekte umzusetzen. Zwar zeigen die Ergebnisse des Krankenhaus Rating Reports aus dem Jahr 2018, dass sich die wirtschaftliche Lage deutscher Krankenhäuser im Jahr 2016 verbessert hat. Trotzdem bleibt die Kapitalausstattung der Krankenhäuser weiterhin unzureichend (Augurzky et al. 2018b). Zu dieser Feststellung passt, dass gemäß einer Studie der Roland Berger Stiftung aus dem Jahr 2017 knapp 60 Prozent der Kliniken von sich selbst sagen, nicht in ausreichendem Maß zu investieren (Roland Berger Stiftung 2017). Gründe dafür sind bei fast allen Kliniken (90\%) die zu geringen Fördermittel und bei 53 Prozent der Befragten zu wenig Einnahmen aus dem Krankenhausbetrieb. Für IT investieren die Kliniken demnach weniger als 2 Prozent ihres Umsatzes. Größte Hürde für die Digitalisierung im Krankenhaus sei laut Aussage der Studienteilnehmer der dafür hohe Investitionsbedarf.

Neben der Frage der Finanzierung stellt sich außerdem die Frage, wie das digitale Krankenhaus der Zukunft konkret aussehen solle (Krüger-Brand 2017). So hat beispielsweise das Fraunhofer-Innovationszentrum für Logistik und IT untersucht, inwiefern der Kerngedanke von Industrie 4.0, die reale mit der virtuellen Welt zu vernetzen, auf ein Krankenhaus übertragbar ist (Fraunhofer-Institut für Materialfluss und Logistik 2017). Das Krankenhaus 4.0 als Zukunftsvision umfasse den Autoren zufolge „die Digitalisierung und Vernetzung von Behandlungs- und Versorgungsprozessen im Krankenhaus mithilfe von „cyberphysikalischen“ Systemen, dem Internet der Dinge und Diensten als Unterstützungssysteme“ (Fraunhofer-Institut für Materialfluss und Logistik 2017; Krüger-Brand 2017). Erste Schritte auf dem Weg zu diesem Ziel sind beispielsweise die EPA, die Aufnahme und Verarbeitung medizinischer Daten über mobile Endgeräte oder die automatisierte fallbasierte Materialerfassung. Für die sukzessive Umsetzung sei ein strategisches Konzept ebenso wie eine fundierte Finanzierung der Investitionen notwendig.

\subsection{Kann Deutschland von Dänemark lernen?}

Die aktuelle dänische Krankenhauslandschaft ist geprägt von einem drastischen strukturellen Wandel, der von politischer Seite systematisch vorangetrieben und in vielfacher Hinsicht als vorbildlich angesehen wird. Noch bis Ende der 1990er Jahre waren kleine regionale Allgemein-Krankenhäuser in großer Anzahl über das gesamte Land verteilt. Aufgrund einer Strukturreform, bei der vor allem die Qualität der Patientenversorgung und weniger die hohen Vorhaltekosten für die Krankenhauskapazitäten im Vordergrund standen, wurden seit der Jahrtausendwende eine Vielzahl dieser kleinen Krankenhäuser geschlossen und die Anzahl der Bettenkapazitäten deutlich reduziert. Es fand in Dänemark eine Abkehr von der wohnort-zentrierten Krankenhausversorgung hin zu einer spezialisierten Versorgung mit starker intersektoraler Vernetzung und einer Restrukturierung der Notfallversorgung statt. Gegenwärtig weist Dänemark eine der niedrigsten Bettendichten (2014: 2,5 Betten pro 1.000 Einwohner) und kürzesten Verweildauern (2014: 3,5 Tage) in Europa auf (OECD 2016) und beide Kennziffern sollen zukünftig sogar weiter deutlich reduziert werden (Danish Ministry of Health 2016).

Im Gegensatz zu Deutschland hat Dänemark ein staatliches, steuerfinanziertes Gesundheitssystem, bei dem sowohl die Planung und Trägerschaft als auch die Vergütung der Krankenhäuser in staatlicher Verantwortung liegen. Somit stammen sowohl die Behandlungsvergütung über das Fallpauschalensystem als auch die Investitionsfinanzierung über nationale und regionale Fördermittel direkt aus dem Staatshaushalt. Die Zuständigkeit für die Krankenhäuser liegt bei den fünf dänischen Regionen, die allerdings keine politische Eigenständigkeit besitzen, sondern vielmehr verwaltungstechnische Einheiten zwischen Zentralverwaltung und den Kommunen bilden. Die übergeordnete politische Steuerung und Koordination der Krankenhäuser, speziell hinsichtlich der Spezialisierung von Leistungen, findet durch die nationale Regierung statt.

Seit der Gesundheitsreform im Jahr 2007 plant die dänische Gesundheitsbehörde (DHMA) für insgesamt 36 spezialisierte Leistungsbereiche die 
regionale Verteilung dieser Angebote auf die Krankenhäuser. Diese erfolgt in Abhängigkeit der Komplexität, der Häufigkeit und des Ressourcenaufwands der Leistungen und anhand eines einheitlichen Kriterienkatalogs, um die Qualität der Leistungserbringung (z. B. über Mindestmengen) sicherzustellen (Danish Ministry of Health 2016). Grundsätzlich sollten spezialisierte Leistungen in ein bis drei Krankenhäusern je Region und hochspezialisierte Behandlungen in ein bis drei Krankenhäusern landesweit angeboten werden. Für besonders seltene Leistungen ist sogar die Behandlung im Ausland anstatt eines eigenen Angebots in dänischen Krankenhäusern vorgesehen.

Mit einem groß angelegten nationalen Investitionsprogramm („Danish Super Hospital Program“) wird die Krankenhausstruktur in Dänemark aktuell weiter zentralisiert und modernisiert. Bis 2022 wird es landesweit insgesamt 16 so genannte Superkrankenhäuser geben, wovon sieben dieser Häuser als komplette Neubauten geplant wurden. Sie sollten im Zeitraum zwischen 2017 und 2022 einsatzbereit sein. Die neun anderen Superkrankenhäuser werden oder wurden an bestehenden Standorten erweitert und modernisiert. Die gesamte Investitionssumme für dieses Strukturprogramm beträgt rund 5,6 Mrd. Euro und damit etwa 1.000 Euro pro Einwohner. Die Mittel stammen im Wesentlichen aus nationalen Fördermitteln (60 \%) und den zugewiesenen Regionalbudgets (27,5\%). Darüber hinaus soll auch der Grundstücksverkauf von dann nicht mehr benötigten Krankenhausstandorten in nicht unerheblichem Umfang (12,5 \%) zur Finanzierung des Programms beitragen.

Die Auswahl der Investitionsprojekte fand auf regionaler Ebene in Abstimmung mit der Zentralregierung und unter Berücksichtigung einheitlicher Kriterien statt. Dabei erfolgte die Zusage der staatlichen Fördermittel für die ausgewählten Investitionsprojekte nur im Gegenzug zur Bereitschaft der Regionen zu generellen Strukturanpassungen mit Schließung von kleinen lokalen Krankenhäusern. Die Regionen sind individuell verantwortlich für die Errichtung der Superkrankenhäuser in ihrem Gebiet und müssen gegenüber der Zentralregierung regelmäßig Rechenschaft über den Fortschritt und die Einhaltung der fix vorgegebenen Budgets für jedes einzelne Projekt ablegen.
Im Gegenzug zu den hohen Investitionen erwartet die dänische Regierung von den Regionen deutliche Effizienzgewinne durch den Betrieb der modernen Superkrankenhäuser in Höhe von 4 bis 8 Prozent gegenüber dem vorherigen Status quo $^{3}$. Erreicht werden soll dies vor allem über

- den Abbau von Bettenkapazitäten,

- die Zusammenlegung und Restrukturierung von Krankenhäusern und Versorgungszentren,

- den Ausbau von ambulanter bzw. intersektoraler Versorgung,

- den verstärkten Einsatz von modernen IT- und Logistiklösungen.

Dänemark gilt schon seit langem als Vorreiter in der Nutzung von E-Health-Lösungen, wie z. B. der elektronischen Patientenakte (EPA) oder elektronischen Überweisungen und Arzneimittelverschreibungen (Currie und Seddon 2014). Im Rahmen der Krankenhausneustrukturierung wird diesem Bereich ebenfalls ein besonderer Stellenwert beigemessen, indem ein festgeschriebener Anteil von 20 Prozent der gesamten Investitionssumme explizit für den Bereich IT und Logistik vorgesehen ist. Damit sollen die organisatorischen und medizinischen Abläufe innerhalb des Krankenhauses erheblich optimiert und effizienter gestaltet werden. Außerdem sollen die telemedizinischen Anbindungen außerhalb der Klinik deutlich verbessert werden, um durch einen reibungslosen Informationsaustausch der Krankenhäuser mit niedergelassenen Ärzten, Rettungsdienst, Pflegeeinrichtungen und direkt mit den Patienten die Zahl der Krankenhausfälle und die stationäre Verweildauer weiter zu reduzieren. So sollen bis zum Jahr 2020 folgende Ziele erreicht werden:

- Steigerung der Zahl der ambulanten Behandlungen um 50 Prozent,

- Abbau der Bettenkapazitäten um 20 Prozent und

- Reduzierung der stationären Verweildauer auf ca. drei Tage.

3 Die Regierung erwartet Einsparungen durch die Effizienzgewinne in Höhe von rd. 300 Mio. Euro pro Jahr, sodass sich die Gesamtinvestitionssumme unter der hypothetischen Annahme ansonsten gleichbleibender Rahmenbedingungen nach 20 Jahren amortisiert hätte. 
Mit der massiven Umstrukturierung des Krankenhauswesens zeigt das Gesundheitsministerium in Dänemark vorbildlich, wie eine Reform zu effizienten Strukturen in der stationären Versorgung angegangen werden kann ( $\triangleright$ Kapitel 7 im vorliegenden Band). Aufbauend auf einer umfassenden Bestandsaufnahme mit klar definierten Zielen, der Bereitschaft zur Schließung vieler kleiner Einheiten und der Bereitstellung von enormen Investitionsmitteln soll die Versorgungssituation für die dänische Bevölkerung verbessert werden. Dabei steht die Qualität der Versorgung im Vordergrund, aber gleichzeitig sollen durch moderne Krankenhausstrukturen auch erhebliche Effizienzsteigerungen ermöglicht werden. Wichtig sind neben der architektonischen und organisatorischen Gestaltung bei Krankenhausneubauten vor allem auch die enge Vernetzung zwischen dem stationären Sektor und dem niedergelassenen Bereich sowie die starke Einbeziehung von modernen IT- und E-Health-Lösungen bereits bei der Planung und Bereitstellung von Fördermitteln.

Darüber hinaus zeigt das Beispiel Dänemark im Bereich der Notfallversorgung, dass eine flächendeckende Sicherstellung auch bei einer starken Konzentration und Zentralisierung der Krankenhauslandschaft gewährleistet werden kann. Die Anzahl der Notaufnahmen wird von 40 auf nur noch 20 Einrichtungen halbiert und gleichzeitig werden strukturelle Anpassungsmaßnahmen getroffen, um die Versorgungsqualität trotz steigender Entfernungen zu verbessern (Augurzky et al. 2015). Trotz existierender Versorgungsunterschiede zwischen den Ländern kann das Beispiel Dänemark für die deutsche Gesundheitspolitik Wege aufzeigen, wie strukturelle Anpassungen und Investitionen im Krankenhauswesen generell angegangen werden können, auch wenn die Rahmenbedingungen in beiden Ländern in wesentlichen Bereichen sehr große Unterschiede aufweisen. So existieren in Dänemark keine eigenständigen Bundesländer, keine Krankenversicherungen, keine Kassenärztlichen Vereinigungen mit der ambulant-stationären Sektorengrenze und keine unterschiedlichen Krankenhausträgerschaften. Nichtsdestotrotz ließen sich in manchen Regionen Deutschlands durchaus moderne integrierte „Superkrankenhäuser“ errichten und auf Bundes- und Landesebene könnte die
Digitalisierung der Krankenhaus- und generell der Gesundheitsversorgung ähnlich wie in Dänemark gefördert werden.

\subsection{Ansätze zur gelenkten Investi- tionsfinanzierung im Bereich der Digitalisierung: Der „Digital Boost"}

Für eine breit angelegte Digitalisierungsstrategie des deutschen Krankenhausmarktes braucht es ausreichend Investitionskapital, welches aufgrund der bestehenden Förderlücke nicht zur Verfügung steht. Der aktuelle Krankenhaus Rating Report 2018 schätzt die Förderlücke auf etwa 3,0 Mrd. Euro jährlich (Augurzky et al. 2018). Zum Teil schließen die Krankenhäuser diese jährliche Investitionslücke aus eigener Kraft und versuchen auch Investitionen im Bereich der Digitalisierung selbst zu finanzieren. Jedoch gelingt es den Kliniken nicht, die Förderlücke gänzlich zu schließen, sodass es $\mathrm{zu}$ einem schleichenden Substanzabbau und zu einem zu geringen Digitalisierungsaufbau kommt (Augurzky et al. 2018b).

Vor diesem Hintergrund hat das RWI im Auftrag des Bundesministeriums für Gesundheit ein Gutachten zum „Stand und Weiterentwicklung der Investitionsförderung im Krankenhausbereich" erstellt (Augurzky et al. 2017) und dabei verschiedene Vorschläge erarbeitet, um die bestehende Förderlücke zu beheben. Dabei erfolgt auch ein internationaler Vergleich zur Investitionsfinanzierung von Krankenhäusern. Ein Fokus wurde dabei auf die Möglichkeiten der Finanzierung des „digitalen Wandels" der deutschen Krankenhäuser gelegt. Konkret wird ein so genannter Digital Boost vorgeschlagen. Dieser sieht vor, Investitionen in die Digitalisierung gezielt durch ein zeitlich befristetes Investitionsprogramm von acht Jahren zu fördern. Ziel ist, eine zeitgemäße IT-Infrastruktur und die elektronische Vernetzung der Krankenhäuser zu ermöglichen, wie sie manch anderen Ländern bereits existiert.

Wie oben erwähnt, sieht Dänemark vor, dass 20 Prozent der Investitionen in neue Krankenhausstrukturen für IT und Logistik zur Verfügung stehen sollen (Danish Ministry of Health 2016). Be- 
Zeitlich befristetes Investitionsprogramm (z.B. 8 Jahre) von 720 Mio. $€$ p.a. Bundesmittel mit Ko-Finanzierung der Länder $+50 \%$ (on top ihres heutigen Fördervolumens) für Auf- und Ausbau moderner Technologien in KH (z.B. IT-Infrastruktur, Telemedizin, Robotik, Interoperabilität, digitale Interaktion mit Patienten)(1)

\begin{tabular}{|c|c|c|}
\hline & Ziele? & Reduktion der Investitionslücke im Bereich „Digitalisierung“ \\
\hline 2 & $\begin{array}{r}\text { Art von } \\
\text { Investitionen? }\end{array}$ & $\begin{array}{l}\text { Digitale Infrastruktur für betriebsübergeordnete Aufgaben gemäß Kriterienkatalog } \\
\text { Nicht für allgemeine IT-Ausstattung der KH }\end{array}$ \\
\hline 3 & Für wen? & Alle Plankrankenhäuser \\
\hline 4 & Wie verteilen? & $\begin{array}{l}\text { Auf Antrag, bei Erfüllung der Kriterien mit gleichen Chancen für alle Krankenhäuser - aber mit } \\
\text { struktureller Komponente (z.B. Vorrang für Breitbandanschluss / Telemedizin in ländlichen Regionen) }\end{array}$ \\
\hline & Wie lange? & Begrenzt, z.B. auf 8 Jahre \\
\hline 6 & Wer finanziert? & Mittel des Bundes und der Länder \\
\hline 7 & Voraussetzung? & Bundesweit einheitlicher Kriterienkatalog \\
\hline 8 & Nebeneffekte? & $\begin{array}{l}\text { Bei unscharfer Definition der Kriterien, mögliche Mitnahmeeffekte der Krankenhäuser bei } \\
\text { IT-Investitionen zur Reduktion der Betriebskosten }\end{array}$ \\
\hline & $\begin{array}{r}\text { Rechtliche } \\
\text { Umsetzbarkeit? }\end{array}$ & Ja, soweit keine vorrangigen Regelungen bestehen \\
\hline
\end{tabular}

Krankenhaus-Report 2019

- Abb. 5.3 Digital Boost (Daten aus Augurzky et al. 2017)

zogen auf den jährlichen Investitionsbedarf von 5,4 Mrd. Euro für Deutschland wären dies 1,08 Mrd. Euro. Wenn der Bund die Digitalisierung im Krankenhausbereich direkt fördern möchte, so könnte er dazu 720 Mio. Euro jährlich beitragen, ergänzt um eine Ko-Finanzierung der Länder von 50 Prozent auf die Bundesmittel, d. h.360 Mio. Euro. (im Detail s. dazu auch Augurzky et al. 2017). Die Mittel würden nur für den Ausbau der digitalen Infrastruktur der Plankrankenhäuser bereitgestellt (- Abb. 5.3). Dabei ist Voraussetzung, dass die Länder ihr bisheriges Fördermittelvolumen nicht absenken. Bei einem Förderzeitraum von beispielsweise acht Jahren ergäben sich insgesamt 8,6 Mrd. Euro zusätzliche Fördermittel. Von Seiten der Krankenhäuser in Deutschland wurde das notwendige Fördervolumen für IT-Investitionen auf rund $11 \mathrm{rd}$. Euro über den Zeitraum der nächsten sieben Jahre geschätzt (VKD 2016).

Das hier vorgeschlagene Förderprogramm zielt speziell auf Digitalisierungsmaßnahmen ab, die einen externen Nutzen generieren, der vom Krankenhaus nicht oder nicht vollständig internalisiert werden kann. Dagegen sollten Maßnahmen, die allein dazu dienen, die internen Betriebsprozesse durch Digitalisierung zu verbessern, nicht durch zusätzliche Investitionsmittel gefördert werden. Eine Förderung von solchen Investitionsmaßnahmen mit einem rein betrieblichen Nutzen würde zu Wettbewerbsverzerrungen führen, weil sie diejenigen Krankenhäuser benachteiligt, die in der Vergangenheit aus Eigenmitteln solche Investitionen bereits durchgeführt haben.

Unter die zu fördernde digitale Infrastruktur für betriebsübergeordnete Aufgaben sollten beispielweise die Ausstattung für telemedizinische Maßnahmen, die Schaffung von Interoperabilität bei elektronischem Datenaustausch oder die digitale Interaktion mit Patienten fallen. Zusätzlich könnten auch neuartige Risikoprojekte ${ }^{4}$ gefördert werden, deren interner Nutzen nur mit einer gewissen Wahrscheinlichkeit realisiert werden kann. Zur Abgrenzung der förderfähigen von nicht-förderfähigen Maßnahmen im Bereich der Digitalisierung ist ein bundesweit einheitlicher Kriterienkatalog zu

4 Hierbei ist eine Abgrenzung zur Förderung durch den Innovationsfonds zu beachten. 
erarbeiten. Nach Experteneinschätzung ${ }^{5}$ ist eine Förderung vor allem für folgende Bereiche der Digitalisierung notwendig und sinnvoll:

- Bereitstellung und Betrieb von BreitbandInternetzugängen für Krankenhäuser. In der Regel ist derzeit eine Anschluss-Bandbreite mit mindestens $100 \mathrm{Mbit} / \mathrm{s}$ für aktuelle Anwendungen erforderlich. Dies stellt einen nicht unerheblichen Kostenfaktor für die Krankenhäuser dar. Daher sollte diskutiert werden, ob die Finanzierung der entstehenden Kosten für die Bereitstellung unabhängig von Krankenhausinvestitionsförderprogrammen oder im Rahmen von allgemeinen Fördertöpfen für Infrastrukturmaßnahmen finanziert werden sollen.

- Apparative Ausstattung im Krankenhaus für telemedizinische Anbindung an spezialisierte Versorgungszentren und integrierte Versorgungskonzepte.

- Aufbau einer einheitlichen Telematikinfrastruktur zur besseren Vernetzung und für die Interoperabilität im Rahmen des E-HealthGesetzes. Auch wenn der Telematikzuschlag nach $\$ 291$ Abs. 7a SGB V bereits einen ersten, wichtigen Finanzierungsaufschlag bietet, zeigt die Empirie, dass insbesondere die Interoperabilität noch nicht gegeben ist und v. a. die klinischen Prozesse und die Investition in Humankapital nicht ausreichend (finanziert) sind, um von einer einheitlichen Telematikversorgungsstruktur sprechen zu können.

- Erhöhung der IT-Sicherheit für Krankenhäuser, die zur „kritischen Infrastruktur“ (nach $\S 8$ a BSIG) zählen, z. B. technische Datensicherungssysteme, IT-Sicherheitsmanagement nach ISO 27001 etc. Dies betrifft etwa 80 bis 110 Krankenhäuser in Deutschland. ${ }^{6}$

Vorrang sollten dabei Anträge zur besseren Anbindung von ländlichen Regionen an Zentren haben, zum Beispiel über Breitbandanschluss und Aufbau von Telemedizin. Bei unscharfer Abgrenzung der

5 Angaben von Prof. Dr. med. Siegfried Jedamzik (TH Deggendorf, Bayerische Telemed-Allianz).

6 Ggf. wären solche Maßnahmen jedoch vom Bundesministerium des Inneren zu fördern. förderfähigen Maßnahmen sind Mitnahmeeffekte der Krankenhäuser für IT-Investitionen zu befürchten, die ausschließlich betriebsinternen Zwecken dienen.

Der „Digital Boost“ kann die gesamte Förderlücke bei Krankenhausinvestitionen nicht schließen. Er entlastet nur bei Investitionen im Bereich der IT und Digitalisierung mit positiven externen Effekten. Die Indikationsqualität kann möglicherweise durch verbesserte telemedizinische Anbindung erhöht werden, ebenso die intersektorale Zusammenarbeit, wenn sich die Interoperabilität erhöht. Durch eine einheitliche Telematikinfrastruktur muss knappes, qualifiziertes Personal in geringerem Maße dezentral vorgehalten werden. Es kann vielmehr in telemedizinischen Zentren zentral gebündelt werden. So können Personalressourcen effizienter eingesetzt werden und ggf. Einsparungen generiert werden. Negative Effekte sind nicht zu erwarten, außer wenn Innovatoren innovative, aber möglicherweise nicht förderfähige Ideen im Bereich der Digitalisierung nicht umsetzen, weil sie stattdessen lieber auf förderfähige Projekte setzen.

Die rechtliche Umsetzbarkeit des zeitlich befristeten Investitionsprogramms für den Auf- und Ausbau moderner Technologien in Krankenhäuser, wie beispielsweise die IT-Infrastruktur, die Robotik, die digitale Interaktion mit Patienten u. ä., ist grundsätzlich leichter als in anderen Bereichen. So sieht Art. 91c GG vor, dass der Bund und die Länder bei Planung, Errichtung und Betrieb der für ihre Aufgabenerfüllung benötigten informationstechnischen Systeme zusammenwirken. Die Norm soll eine verfassungsrechtliche Grundlage für die $\mathrm{Zu}$ sammenarbeit von Bund und Ländern in der Informationstechnik schaffen (Maunz und Dürig 2017). Allerdings ist die Beurteilung der rechtlichen Umsetzbarkeit stets davon abhängig, ob bereits vorrangige Regelungen bestehen. So bestehen für den Bereich der Telematik bereits die Sonderzuweisungen nach $₫ 291$ a Abs. 7 SGB V, $\$ 2$ Nr. 2 KHG. Hier wäre also bei der Konkretisierung der Fördermaßnahmen darauf $\mathrm{zu}$ achten, welche Informationstechnologien dies betreffen soll. 


\subsection{Fazit}

Die geburtenstarken Jahrgänge werden schon bald aus dem Erwerbsleben ausscheiden. Der große „Rentenansturm“ ist im Laufe der 2020er Jahre zu erwarten und mithin auch eine wachsende Nachfrage nach Gesundheitsleistungen. Im Gegenzug kommen weit weniger jüngere Menschen nach, welche die Arbeit der ausscheidenden Babyboomer übernehmen könnten, sodass mit einem wachsenden Engpass an Fachkräften auf dem Arbeitsmarkt, insbesondere im Gesundheitswesen, zu rechnen ist. Die Gesundheitsbranche muss dann massiv Arbeitskräfte aus anderen Branchen für sich gewinnen, was sehr wettbewerbsfähige Arbeitsplätze verlangt, also u. a. steigende Löhne. Da im Gesundheitswesen die Preise für Gesundheitsleistungen staatlich reguliert sind, können höhere Löhne jedoch nicht einfach über höhere Preise der Leistungserbringer aufgefangen werden. Wenn die Löhne damit nicht ausreichend steigen, droht eine Rationierung auf dem Arbeitsmarkt für Gesundheitsberufe und infolgedessen auch der Leistungen für Patienten. Zentrale Aufgabe wird es daher sein, mit weniger Fachkräften mehr hilfsbedürftige Menschen zu versorgen, ohne dass die Arbeitsbelastung dieser Fachkräfte so sehr steigt, dass sie am Ende das Interesse am Gesundheitswesen verlieren und in anderen Branchen attraktivere Tätigkeiten suchen. Zentraler Schlüssel dabei ist zum einen, dass gewisse Tätigkeiten, die heute noch vom Menschen erbracht werden zukünftig durch mit künstlicher Intelligenz ertüchtigte Roboter ersetzt werden. Zum anderen kann v. a. eine flächendeckende Telematikinfrastruktur dazu beitragen, dass Fachpersonal nicht mehr dezentral, sondern zentral und somit ressourcenschonender vorgehalten werden kann, bei gleichzeitig steigender Behandlungsqualität. Vor dem Hintergrund knapper Personalressourcen wird es perspektivisch einfach nicht mehr möglich sein, allein über 2.000 Krankenhausstandorte mit adäquat qualifiziertem, medizinisch-pflegerischen Fachpersonal in Mindestbesetzung vorhalten zu können.

Die inzwischen viel gerühmte „Digitalisierung“ könnte diese Aufgabe unterstützen. Hilfreich für das stark regulierte Gesundheitswesen wird dabei die Notsituation sein, die im Laufe der 2020er Jahre entstehen wird. Denn solange die Kassen wie derzeit gefüllt sind, wird das Beharrungsvermögen des Gesundheitswesens mitsamt seinen bestehenden Ineffizienzen obsiegen, weil bestehende Besitzstände finanziell bedient werden können. Relevante Veränderungen, gerade in einem regulierten System, lassen sich nur erreichen, wenn die Ressourcen so knapp werden, dass heilige Kühe geschlachtet werden müssen. Durch eine gezielte, staatliche geförderte Investitionslenkung im Bereich der Digitalisierung der Gesundheitswirtschaft können jedoch schon heute wichtige Grundlagen dafür gelegt werden.

Beispielsweise misst Dänemark im Rahmen seiner groß angelegten Umstrukturierung der Krankenhauslandschaft der Digitalisierung einen besonderen Stellenwert bei, indem ein festgeschriebener Anteil von 20 Prozent der gesamten Investitionssumme explizit für den Bereich IT und Logistik vorgesehen ist. Damit sollen die organisatorischen und medizinischen Abläufe innerhalb des Krankenhauses erheblich optimiert und effizienter gestaltet werden. Außerdem sollen die telemedizinischen Anbindungen außerhalb der Klinik deutlich verbessert werden. Für eine breit angelegte Digitalisierungsstrategie des deutschen Krankenhausmarktes braucht es ebenfalls ausreichend Investitionskapital, das aber aufgrund der bestehenden Förderlücke nicht zur Verfügung steht.

Wir schlagen daher einen „Digital Boost“ vor, der Investitionen in die Digitalisierung durch ein zeitlich befristetes Investitionsprogramm von acht Jahren vorsieht. Dessen Ziel ist es, eine zeitgemäße IT-Infrastruktur und die elektronische Vernetzung der Krankenhäuser zu ermöglichen, wie sie manch anderen Ländern bereits existiert. Bezogen auf den jährlichen Investitionsbedarf von mindestens 5,4 Mrd. Euro für Deutschland und der in Dänemark angewandten Quote von 20 Prozent für Digitales wären dies 1,08 Mrd. Euro p. a. Der Bund könnte dazu 720 Mio. Euro jährlich beitragen, ergänzt um eine Ko-Finanzierung der Länder von 50 Prozent auf die Bundesmittel, d. h. 360 Mio. Euro. Bei einem Förderzeitraum von beispielsweise acht Jahren ergäben sich insgesamt 8,6 Mrd. Euro zusätzliche Fördermittel.

Dabei sollten nur Maßnahmen gefördert werden, die einen externen Nutzen generieren, der vom Krankenhaus nicht oder nicht vollständig internalisiert werden kann. Dagegen sollten Maßnahmen, die dazu dienen, die internen Betriebsprozesse 
durch Digitalisierung zu verbessern, nicht durch zusätzliche Investitionsmittel gefördert werden. Förderfähig sollten beispielweise die Ausstattung für telemedizinische Maßnahmen, die Schaffung von Interoperabilität bei elektronischem Datenaustausch oder die digitale Interaktion mit Patienten fallen. Zusätzlich könnten auch neuartige Risikoprojekte gefördert werden, deren interner Nutzen nur mit einer gewissen Wahrscheinlichkeit realisiert werden kann. Zur Abgrenzung der förderfähigen von nicht-förderfähigen Maßnahmen im Bereich der Digitalisierung ist ein bundesweit einheitlicher Kriterienkatalog zu erarbeiten.

\section{Literatur}

Amelung VE, Chase DP, Urbanski D, Bertram N, Binder S (2016) Die elektronische Patientenakte - Fundament einer effektiven und effizienten Gesundheitsversorgung? Gutachten im Auftrag der Stiftung Münch. medhochzwei, Heidelberg Augurzky B (2018) Digitalisierung der Gesundheitswirtschaft: Warum sie unvermeidlich ist. In: Meier, P-M, Düllings J, Henkel A, Nolte G (Hrsg) Digitale Transformation der Gesundheitswirtschaft: Chancen und Herausforderungen in disruptiven Zeiten. Kohlhammer, Stuttgart, erscheint im 4. Quartal 2018

Augurzky B, Beivers A, Giebner M (2015) Organisation der Notfallversorgung in Dänemark: Lösungsansätze für deutsche Probleme? In: Klauber J, Geraedts M, Friedrich J, Wasem J (Hrsg) Krankenhaus-Report 2015 - Schwerpunkt: Strukturwandel. Schattauer, Stuttgart, S 77-97

Augurzky B, Beivers A, Emde A, Halbe B, Pilny A, Straub N (2017) Stand und Weiterentwicklung der Investitionsförderung im Krankenhausbereich. Gutachten im Auftrag des Bundesministeriums für Gesundheit. RWI LeibnizInstitut für Wirtschaftsforschung, Essen.https://www. bundesgesundheitsministerium.de/fileadmin/Dateien/5_ Publikationen/Ministerium/Berichte/Gutachten_Investitionsfoerderung_Krankenhausbereich.pdf. Zugegriffen: 09. Oktober 2018

Augurzky B, Beivers A, Pilny A (2018a) Krankenhäuser in privater Trägerschaft 2018. Gutachten im Auftrag des Bundesverbandes Deutscher Privatkliniken. RWI Leibniz-Institut für Wirtschaftsforschung, Essen. http://www. rwi-essen.de/media/content/pages/publikationen/ rwi-materialien/rwi-materialien_122.pdf. Zugegriffen: 09. Oktober 2018

Augurzky B, Krolop S, Mensen A, Pilny A, Schmidt CM, Wuckel C (2018b) Krankenhaus Rating Report 2018: Personal - Krankenhäuser zwischen Wunsch und Wirklichkeit. medhochzwei, Heidelberg

Currie WL, Seddon JM (2014) A cross-national analysis of eHealth in the European Union: Some policy and research directions. Information \& Management 51 (6):783-797
Danish Ministry of Health (2016) Healthcare in Denmark - An Overview. Ministry of Health, Kopenhagen, Dänemark. http://www.sum.dk. Zugegriffen: 09. Oktober 2018

DIV-Report (2017) Deutschland intelligent vernetzt. Digitale Gesundheit 2017. Digital-Gipfel Plattform Innovative Digitalisierung der Wirtschaft, Fokusgruppe intelligente Vernetzung

Fraunhofer-Institut für Materialfluss und Logistik IML (2017) Das Positionspapier Krankenhaus 4.0: Industrie $4.0 \mathrm{im}$ Gesundheitswesen. Dortmund

Hübner U, Esdar M, Hüsers J, Liebe J-D, Rauch J, Thye J, Weiß J-P (2018) IT-Report Gesundheitswesen, Schwerpunkt: Wie reif ist die IT in deutschen Krankenhäusern? Befragung der bundesdeutschen Krankenhäuser. Hochschule Osnabrück, Osnabrück. https://www.hs-osnabrueck.de/fileadmin/ HSOS/Homepages/IT-Report_Gesundheitswesen/IT-Report_2018_final.pdf. Zugegriffen: 09. Oktober 2018

Kellermann C (2018) Digitalisierung im Gesundheitswesen am Beispiel des smart Hospital. Bachelorthesis. Hochschule Fresenius, Fachbereich Wirtschaft \& Medien, Betreuer: Beivers A, München. Unveröffentlicht

Krüger-Brand H (2017) Digitalisierung im Krankenhaus: Der Infrastruktur fehlt die Finanzierung. Dtsch Arztebl 114(48):A-2258/B-1896/C-185

Lux T, Breil B (2017) Digitalisierung im Gesundheitswesen zwischen Datenschutz und moderner Medizinversorgung. Zeitgespräch. ZBW - Leibniz-Informationszentrum Wirtschaft

Maunz T, Dürig G (Hrsg) (2017) Grundgesetz - Kommentar. Loseblattsammlung 80. Ergänzungslieferung Stand 06/2017. CH Beck, München

OECD (2016) OECD Health Statistics 2016. Organisation for Economic Co-operation and Development, Paris. http://www.oecd.org/els/health-systems/health-data. htm. Zugegriffen: 09. Oktober 2018

Rebitschek FG, Gigerenzer G, Wagner GG (2017) Kritische Voraussetzungen für ein digitales Gesundheitswesen in Deutschland. Zeitgespräch. ZBW - Leibniz-Informationszentrum Wirtschaft

Roland Berger Stiftung (2017) Roland Berger Krankenhausstudie 2017. Hamburg. https://www.rolandberger.com/ de/Publications/pub_german_hospitals_2017.html. Zugegriffen: 09. Oktober 2018

Schumpeter JA (2018) Kapitalismus, Sozialismus und Demokratie, 9. Aufl. UTB Verlag, Stuttgart

SVR (2017) Für eine zukunftsorientierte Wirtschaftspolitik. Jahresgutachten des Sachverständigenrates zur Begutachtung der gesamtwirtschaftlichen Entwicklung. Wiesbaden

VKD - Verband der Krankenhausdirektoren Deutschlands e. V. (2016) Krankenhausmanager fordern IT-Investitionsoffensive von der Politik - Digitalisierung ist zentrales Zukunftsthema für die Gesundheitsbranche. Pressemitteilung vom 14.11.2016. https://www.vkd-online.de/aktuelles/ pressemitteilung-krankenhausmanager-fordern-itinvestitionsoffensive-von-der-politik2. Zugegriffen: 09. Oktober 2018 
Open Access Dieses Kapitel wird unter der Creative Commons Namensnennung 4.0 International Lizenz(http://creativecommons. org/licenses/by/4.0/deed.de) veröffentlicht, welche die Nutzung, Vervielfältigung, Bearbeitung, Verbreitung und Wiedergabe in jeglichem Medium und Format erlaubt, sofern Sie den/die ursprünglichen Autor(en) und die Quelle ordnungsgemäß nennen, einen Link zur Creative Commons Lizenz beifügen und angeben, ob Änderungen vorgenommen wurden.

Die in diesem Kapitel enthaltenen Bilder und sonstiges Drittmaterial unterliegen ebenfalls der genannten Creative Commons Lizenz, sofern sich aus der Abbildungslegende nichts anderes ergibt. Sofern das betreffende Material nicht unter der genannten Creative Commons Lizenz steht und die betreffende Handlung nicht nach gesetzlichen Vorschriften erlaubt ist, ist für die oben aufgeführten Weiterverwendungen des Materials die Einwilligung des jeweiligen Rechteinhabers einzuholen. 